\title{
The SARS-CoV-2 Vaccine Pipeline: an Overview
}

\author{
Wen-Hsiang Chen ${ }^{1}$ (D) $\cdot$ Ulrich Strych ${ }^{1}$ (D) $\cdot$ Peter J Hotez ${ }^{1}$ (D) $\cdot$ Maria Elena Bottazzi ${ }^{1}$ (D)
}

Published online: 3 March 2020

(C) Springer Nature Switzerland AG 2020

\begin{abstract}
Purpose of Review The goal of this review is to provide a timely overview on efforts to develop a vaccine for the 2019 novel coronavirus SARS-CoV-2, the causative agent of coronavirus disease (COVID-19).

Recent Findings Previous research efforts to develop a severe acute respiratory syndrome coronavirus (SARS-CoV) vaccine in the years following the 2003 pandemic have opened the door for investigators to design vaccine concepts and approaches for the COVID-19 epidemic in China. Both SARS-CoV and SARS-CoV-2 exhibit a high degree of genetic similarity and bind to the same host cell ACE2 receptor. Based on previous experience with SARS-CoV vaccines, it is expected that all COVID-19 vaccines will require careful safety evaluations for immunopotentiation that could lead to increased infectivity or eosinophilic infiltration. Besides this, a COVID-19 vaccine target product profile must address vaccinating at-risk human populations including frontline healthcare workers, individuals over the age of 60 , and those with underlying and debilitating chronic conditions. Among the vaccine technologies under evaluation are whole virus vaccines, recombinant protein subunit vaccines, and nucleic acid vaccines.

Summary Each current vaccine strategy has distinct advantages and disadvantages. Therefore, it is paramount that multiple strategies be advanced quickly and then evaluated for safety and efficacy. Ultimately, the safety studies to minimize undesired immunopotentiation will become the most significant bottleneck in terms of time.
\end{abstract}

Keywords Coronavirus $\cdot$ COVID-19 $\cdot$ Wuhan virus $\cdot$ Receptor binding domain $\cdot$ RBD

In December 2019, a novel coronavirus pneumonia emerged in Wuhan, China, linked initially to animal-to-human transmission in local wet markets. Subsequently, human-to-human transmission of the virus commenced, resulting in widespread respiratory illness in Wuhan and other urban areas of Hubei Province, China. The coronavirus then spread across China and at least 20 other nations [1]. On February 11, the World Health Organization named the virus SARS-CoV-2 and the syndrome was named COVID-19, or coronavirus disease 2019 [2]. Although not as lethal as the severe acute respiratory syndrome (SARS) outbreak in 2003, COVID-19 is still characterized by severe respiratory illness and significant mortality, especially among individuals over the age of 60 years and

This article is part of the Topical Collection on Hot Topics in Tropical Medicine

Maria Elena Bottazzi

bottazzi@bcm.edu

1 Texas Children's Hospital Center for Vaccine Development, Departments of Pediatrics and Molecular Virology \& Microbiology, National School of Tropical Medicine, Baylor College of Medicine, Houston, TX, USA in those with underlying chronic conditions such as diabetes and hypertension [1]. Moreover, SARS-CoV-2 is highly transmissible with an estimated reproductive number $\left(R_{0}\right)$ of 2.2 , i.e., one infected individual is estimated to transmit the virus to 2.2 other individuals, and a mean incubation period of 5.8 days [1]. The finding that SARS-CoV-2 is transmitted from infected individuals without symptoms [3], together with its ability to cause pandemic disease within a period of weeks, suggests that control of this viral infection will be challenging without the prospect of a vaccine. Here we provide a brief overview of some of the major candidates and the challenges of implementing vaccine strategies. Since much of the information about these vaccines has not yet entered the peerreviewed literature, for this brief overview, we rely significantly on the information made publicly available on websites and other documents.

\section{COVID-19 and Target Product Profile}

Like the SARS coronavirus, SARS-CoV-2 is believed to have originated from bats before infecting one or more mammal species sold in Wuhan animal markets. Both coronaviruses 
bind to similar ACE2 receptors found in the human lung [4, 5], and both exhibit genomes of approximately $30 \mathrm{~kb}$ [6]. SARS-CoV-2 exhibits approximately $89 \%$ nucleotide similarly to SARS-like coronaviruses (genus Betacoronavirus) found in Chinese bats [6]. On this basis, the early shaping of potential SARS-CoV-2 vaccine strategies built on those advanced previously for SARS.

One of the major hurdles in the early development of SARS coronavirus vaccines has been the finding of undesired immunopotentiation in the form of eosinophilic infiltration or increased infectivity, which is noted to occur following challenge infections after immunizations with whole virus vaccines or even complete spike protein vaccines [7]. The basis of this finding is still under investigation, but it was not considered surprising given that this phenomenon also occurred with whole virus respiratory syncytial virus (RSV) vaccines [7]. Therefore, any vaccine target product profile (TPP) must give full safety considerations to avoid the immunopotentiation (Box 1).

Another key element of a SARS-CoV or SARS-CoV-2 vaccine TPP is the intended target population. Currently, those at the highest risk of acquiring COVID-19 or suffering significant health deterioration are frontline healthcare workers, individuals over the age of 60 years, or those with underlying diabetes and hypertension [8]. Therefore, such populations might be prioritized for vaccine clinical trials or licensure. Still, another feature is the real possibility that a new vaccine for COVID-19 may not be available in time for this current epidemic or pandemic. Therefore, it should be made available for potential stockpiling. Along those lines, vaccines developed and produced for global health security or biodefense are often considered less profitable than traditional childhood and adult vaccines. For that reason, very few of the major multinational pharmaceutical companies appear to have expressed upfront commercial interest in entering this space.

\section{The Strategies}

Shown in Table 1 is a summary of the major COVID-19 vaccines under development. This list is not exhaustive but instead reflects some of the major vaccines highlighted in company materials or publicly available documents, including biocentury.com [9].

Box 1 Key features of a target product profile for a human COVID-19 vaccine
Whole Virus Vaccines Live-attenuated or inactive whole virus vaccines represent a classic strategy for viral vaccinations. According to an industry newsletter, Johnson \& Johnson is one of the few multinational companies embarking on COVID-19 vaccines [20]; similar to their Ebola vaccine platform, they are employing Janssen's AdVac $®$ adenoviral vector and manufacturing in their PER.C6 $®$ cell line technology [20, 21]. In addition, researchers at the University of Hong Kong have developed a live influenza vaccine that expresses SARS-CoV-2 proteins [22]. Finally, Codagenix has developed a "codon deoptimization" technology to attenuate viruses [23] and is exploring SARS-CoV-2 vaccine strategies. A major advantage of whole virus vaccines is their inherent immunogenicity and ability to stimulate toll-like receptors (TLRs) including TLR 3, TLR 7/8, and TLR 9. However, live virus vaccines often require extensive additional testing to confirm their safety. This is especially an issue for coronavirus vaccines, given the findings of increased infectivity following immunization with live or killed whole virus SARS coronavirus vaccines [7].

Subunit Vaccines Subunit vaccines for both SARS coronaviruses rely on eliciting an immune response against the S-spike protein to prevent its docking with the host ACE2 receptor [7]. Already, under funding from the Coalition for Epidemic Preparedness (CEPI), the University of Queensland is synthesizing viral surface proteins, to present them more easily to the immune system. Moreover, Novavax has developed and produced immunogenic virus-like nanoparticles based on recombinant expression of the S-protein [24] while Clover Biopharmaceuticals is developing a subunit vaccine consisted of a trimerized SARS-CoV-2 S-protein using their patented Trimer-Tag® technology [14], although some full-length S-proteins for SARS also elicit increased infectivity and eosinophilic infiltration. Accordingly, a consortium led by Texas Children's Hospital Center for Vaccine Development at Baylor College of Medicine (including University of Texas Medical Branch and New York Blood Center) has developed and tested a subunit vaccine comprised of only the receptor-binding domain (RBD) of the SARS-CoV S-protein $[7,25,26]$. When formulated on alum, the SARSCoV RBD vaccine elicits high levels of protective immunity on the homologous virus challenge. An advantage of the RBD-based vaccine is its ability to minimize host immunopotentiation [7]. Initial findings that the SARS-CoV and SARS-CoV-2 RBDs exhibit more than $80 \%$ amino acid similarity and bind to the same ACE2 receptor offer an opportunity to develop either protein as a subunit vaccine.

Nucleic Acid Vaccines Several major biotechs have advanced nucleic acid vaccine platforms for COVID-19. For example, Inovio Pharmaceuticals is developing a DNA vaccine, while others, such as Moderna Therapeutics and Curevac, are 
Table 1 Major COVID-19 vaccine development programs

\begin{tabular}{|c|c|c|}
\hline Consortium & Candidate vaccine & Reference \\
\hline \multicolumn{3}{|l|}{ Whole virus vaccines } \\
\hline Janssen (Johnson \& Johnson) & 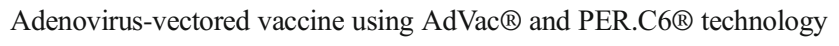 & {$[10]$} \\
\hline Codagenix/Serum Institute of India & Live-attenuated vaccine & {$[11]$} \\
\hline \multicolumn{3}{|l|}{ Subunit vaccines } \\
\hline University of Queensland/CEPI & Protein-based vaccine using Molecular Clamp platform & {$[12]$} \\
\hline Novavax & Recombinant nanoparticle technology & [13] \\
\hline Clover Bipharmaceuticals & S-trimer recombinant protein using Trimer-Tag technology & {$[14]$} \\
\hline $\begin{array}{l}\text { Baylor College of Medicine, Fudan University, } \\
\text { New York Blood Center, Univ Texas Medical Branch }\end{array}$ & Coronavirus RBD protein-based vaccine & {$[15]$} \\
\hline Vaxart & Oral recombinant protein vaccine using VAAST platform & {$[16]$} \\
\hline \multicolumn{3}{|l|}{ Nucleic acid vaccines } \\
\hline Inovio/Beijing Advaccine Biotechnology Co./CEPI & DNA vaccine (INO-4800, based on INO-4700 MERS vaccine) & {$[17]$} \\
\hline Moderna/NIH/CEPI & mRNA vaccine & {$[18]$} \\
\hline CureVac/CEPI & mRNA vaccine & [19] \\
\hline
\end{tabular}

exploring RNA vaccine platforms. The concept of immunizing with DNA began with promising results in mice in 1993 showing protective immunity against influenza, but for decades, these findings have not translated to similar findings in humans. More recently, new modifications and formulations have improved nucleic acid performance in humans, with an expectation that this approach might eventually lead to the first licensed human nucleic acid vaccine.

\section{Concluding Statement}

There are now at least a half-dozen candidates, including live viruses, recombinant protein subunits, and nucleic acids that may ultimately offer promise as preventive vaccines against COVID-19. However, each of these vaccines may require additional manufacturing steps and formal toxicology testing before submitting a regulatory package to national regulatory agencies and be able to commence the clinical development, first with phase 1 clinical trials for safety and immunogenicity, and later, phase 2 and phase 3 trials for both safety and efficacy. The next few months represent a critical period for determining whether the COVID-19 epidemic in Wuhan and Central China will spread across Asia and globally, or whether the warmer months of spring and summer in the Northern Hemisphere will reduce transmission of typically seasonal coronavirus infections. In the former situation, we will urgently require new vaccines. However, the challenge remains. Moving forward, we must recognize that we have had a new major coronavirus epidemic every decade in the twenty-first century-SARS in the 2000s, MERS in the 2010s, and now COVID-19. It is, therefore, a global security priority to advance and stockpile coronavirus vaccines, and to identify international funding mechanisms to support their development, manufacture, and storage. As a matter of fact, this recent outbreak should serve as a beacon to call upon the international research community to not only react but also prepare for the next coronavirus crossing into mammals. A pancoronavirus vaccine is urgently needed and appears scientifically feasible if sufficient resources are made available in due time.

\section{Compliance with Ethical Standards}

Conflict of Interest The authors have developed subunit vaccines against SARS and MERS coronavirus infections. They are involved in the process of developing a vaccine against SARS-CoV-2.

\section{References}

Papers of particular interest, published recently, have been highlighted as:

- Of importance

•- Of major importance

1. Li Q, Guan X, Wu P, Wang X, Zhou L, Tong Y, et al. Early transmission dynamics in Wuhan, China, of novel coronavirus-infected pneumonia. N Engl J Med. 2020. https://doi.org/10.1056/ NEJMoa2001316.

2. World Health Organization. WHO Director-General's remarks at the media briefing on 2019-nCoV on 11 February 2020. 2020. https://www.who.int/dg/speeches/detail/who-director-general-sremarks-at-the-media-briefing-on-2019-ncov-on-11-february2020. Accessed February 13, 2020.

3. Rothe C, Schunk M, Sothmann P, Bretzel G, Froeschl G, Wallrauch C, et al. Transmission of 2019-nCoV infection from an asymptomatic contact in Germany. N Engl J Med. 2020. https://doi.org/10. 1056/NEJMc2001468. 
4. Hoffmann M, Kleine-Weber H, Krüger N, Müller M, Drosten C, Pöhlmann S. The novel coronavirus 2019 (2019-nCoV) uses the SARS-coronavirus receptor ACE2 and the cellular protease TMPRSS2 for entry into target cells. bioRxiv. 2020: 2020.01.31.929042. doi:https://doi.org/10.1101/2020.01.31. 929042.

5. Zhou P, Yang X-L, Wang X-G, Hu B, Zhang L, Zhang W et al. Discovery of a novel coronavirus associated with the recent pneumonia outbreak in humans and its potential bat origin. bioRxiv. 2020:2020.01.22.914952. doi:https://doi.org/10.1101/2020.01.22. 914952.

6. Wu F, Zhao S, Yu B, Chen Y-M, Wang W, Hu Y et al. Complete genome characterisation of a novel coronavirus associated with severe human respiratory disease in Wuhan, China. bioRxiv. 2020:2020.01.24.919183. doi:https://doi.org/10.1101/2020.01.24. 919183.

7. Jiang S, Bottazzi ME, Du L, Lustigman S, Tseng CT, Curti E, et al. Roadmap to developing a recombinant coronavirus $\mathrm{S}$ protein receptor-binding domain vaccine for severe acute respiratory syndrome. Expert Rev Vaccines. 2012;11(12):1405-13. https://doi.org/ 10.1586/erv.12.126.

8. Huang C, Wang Y, Li X, Ren L, Zhao J, Hu Y, et al. Clinical features of patients infected with 2019 novel coronavirus in Wuhan, China. Lancet. 2020. https://doi.org/10.1016/S01406736(20)30183-5.

9. Pong W. A dozen vaccine programs under way as WHO declares coronavirus public health emergency. Biocentury. 2020. https:// www.biocentury.com/article/304328/industry-and-academiccenters-are-rushing-to-create-new-vaccines-and-therapeuticstargeting-coronavirus. Accessed $28 \mathrm{Feb} 2020$.

10. Novel Coronavirus. @jnjnews. 2020. https://www.jnj.com/ coronavirus. Accessed February 10, 2020.

11. BioSpace. Codagenix and Serum Institute of India Initiate CoDevelopment of a Scalable, Live-Attenuated Vaccine Against the 2019 Novel Coronavirus, COVID-19. 2020. https://www.biospace. com/article/releases/codagenix-and-serum-institute-of-indiainitiate-co-development-of-a-scalable-live-attenuated-vaccineagainst-the-2019-novel-coronavirus-covid-19/. Accessed 2/13/ 2020.

12. Hennessy J. Australia's been asked to make a coronavirus vaccine at 'unprecedented speed'. Science Alert. 2020. https://www. sciencealert.com/australian-scientists-asked-to-make-coronavirusvaccine-at-unprecedented-speed. Accessed 28 Feb 2020.

13. Pharmaceutical Technology N. Coronavirus: Vir Biotechnology and Novavax announce vaccine plans. 2020. https://www. pharmaceutical-technology.com/news/coronavirus-virbiotechnology-novavax-vaccine/. Accessed 28 Feb 2020.

14. Clover Biopharmaceuticals. Clover initiates development of recombinant subunit-trimer vaccine for Wuhan coronavirus (2019nCoV). 2020.

15. Mukherjee $\mathrm{S}$. The first coronavirus drug candidate is set for testing in China. Fortune. 2020. https://fortune.com/2020/02/03/ coronavirus-vaccine-testing-in-china/. Accessed 28 Feb 2020.
16. Vaxart. PipelineReview.com Vaxart Announces Initiation of Coronavirus Vaccine Program. 2020. https://pipelinereview.com/ index.php/2020020273689/Vaccines/Vaxart-Announces-Initiationof-Coronavirus-Vaccine-Program.html. Accessed 28 Feb, 2020.

17. Inovio Pharmaceuticals. Inovio Collaborating With Beijing Advaccine To Advance INO-4800 Vaccine Against New Coronavirus In China. 2020. http://ir.inovio.com/news-and-media/ news/press-release-details/2020/Inovio-Collaborating-WithBeijing-Advaccine-To-Advance-INO-4800-Vaccine-AgainstNew-Coronavirus-In-China/default.aspx. Accessed 28 Feb 2020.

18. Park A. Inside the Company That's Hot-wiring Vaccine Research in the Race to Combat the Coronavirus. Time. 2020. https://time.com/ 5775784/coronavirus-vaccine-research/. Accessed 28 Feb 2020.

19. Smith J. CureVac bids to develop first mRNA coronavirus vaccine. 2020. https://www.labiotech.eu/medical/curevac-coronavirusoutbreak-cepi/. Accessed 28 Feb 2020.

20. J\&J working on coronavirus vaccine. thepharmaletter 2020. https:// www.thepharmaletter.com/article/j-j-working-on-coronavirusvaccine. Accessed 28 Feb 2020.

21. ClinicalTrials.gov. National Institutes of Health. https:// clinicaltrials.gov/ct2/show/NCT02543567. Accessed February 7, 2020.

22. Cheung E. China coronavirus: Hong Kong researchers have already developed vaccine but need time to test it, expert reveals. South China Morning Post. https://www.scmp.com/news/hong-kong/ health-environment/article/3047956/china-coronavirus-hongkong-researchers-have. Accessed 28 Feb 2020.

23. Shieber J. Codagenix raises $\$ 20$ million for a new flu vaccine and other therapies. Tech Crunch. https://techcrunch.com/2020/01/13/ codagenix-raises-20-million-for-a-new-flu-vaccine-and-othertherapies/. Accessed 28 Feb 2020.

24. Coleman CM, Liu YV, Mu H, Taylor JK, Massare M, Flyer DC, et al. Purified coronavirus spike protein nanoparticles induce coronavirus neutralizing antibodies in mice. Vaccine. 2014;32(26): 3169-74. https://doi.org/10.1016/j.vaccine.2014.04.016.

25. Chen WH, Chag SM, Poongavanam MV, Biter AB, Ewere EA, Rezende W, et al. Optimization of the production process and characterization of the yeast-expressed SARS-CoV recombinant receptor-binding domain (RBD219-N1), a SARS vaccine candidate. J Pharm Sci. 2017;106(8):1961-70. https://doi.org/10.1016/j. xphs.2017.04.037.

26. Chen WH, Du L, Chag SM, Ma C, Tricoche N, Tao X, et al. Yeastexpressed recombinant protein of the receptor-binding domain in SARS-CoV spike protein with deglycosylated forms as a SARS vaccine candidate. Hum Vaccines Immunother. 2014;10(3):64858 .

Publisher's Note Springer Nature remains neutral with regard to jurisdictional claims in published maps and institutional affiliations. 